\title{
Resection of Fibrous Dysplasia of the Sphenoid Bone and a Concomitant Calcified Pituitary Adenoma via an Endoscopic Endonasal Transsphenoidal Approach: Case Report
}

\author{
Benedict Tan, BS', Sanjay Yadla, MD², Peter G. Campbell, MD² \\ Marc R. Rosen, MD², James J. Evans, MD² \\ 'Jefferson Medical College, Thomas Jefferson University, Philadelphia, PA 19107 USA \\ 2Department of Neurological Surgery, Thomas Jefferson University, Philadelphia, PA 19107 USA \\ 2Department of Otolaryngology, Head \& Neck Surgery, Thomas Jefferson University, \\ Philadelphia, PA 19107 USA
}

\section{Abstract}

Background: Although pituitary adenomas have been described in association with polyostotic fibrous dysplasia in McCune-Albright Syndrome, no such relationship has been described with monostotic fibrous dysplasia. The authors describe a case of monostotic fibrous dysplasia of the sphenoid bone and concomitant pituitary adenoma in a 25 -year-old male. To the authors' knowledge this is the first such case reported in the literature. Clinical presentation, pathology, and surgical approach are described in detail.

Case Description: A 25-year-old male initially presented with headaches, gynecomastia, and galactorrhea. Magnetic Resonance Imaging (MRI) revealed a sellar/suprasellar mass possibly consistent with a calcified pituitary adenoma or craniopharyngioma and a cranial base lesion obstructing the sphenoid sinus consistent with fibrous dysplasia. Both lesions were accessible via an endoscopic transnasal approach. The patient underwent resection of the affected sphenoid bone which allowed appropriate exposure for subsequent resection of the pituitary lesion without major complication. Pathology and immunohistochemical studies confirmed the diagnoses and postoperative imaging revealed gross total resection of the adenoma.

Conclusions: The authors describe a case of monostotic fibrous dysplasia of the sphenoid bone and pituitary adenoma. Resection of such dual lesions can be both safe and efficacious via an endoscopic endonasal transsphenoidal approach.

Key Words: Fibrous Dysplasia, Calcified Pituitary Adenoma, Pituitary Stone, Transsphenoidal

Abbreviations List: MRI: Magnetic Resonance Imaging, CT: Computerized Tomography, CAM: Cell Adhesion Molecule, EMA: Ethidium Moazide, TSH: Thyroid Stimulating Hormone

\section{Case Report}

History and Physical

A 25-year-old African-American male presented with complaints of headaches and galactorrhea. There was no history of long bone fractures. On exam, the patient was noted to have intermittent esotropia of the left eye and gynecomastia. No café au lait spots or other skin lesions were noted. Humphrey visual field testing was normal. Endocrine evaluation revealed a low testosterone level and slightly elevated prolactin level.

\section{Radiology}

CT and MRI of the brain and skull were performed. A $1.4 \mathrm{~cm}$ calcified sellar/suprasellar mass was noted (Figure 1). This lesion extended superiorly to abut the left optic chiasm and optic nerve. Pituitary adenoma or craniopharyngioma were suspected based on these findings. Additionally, there was opacification of the left sphenoid sinus with hypointense material seen on both precontrast $\mathrm{T} 1$ and T2 weighted MRI. This material enhanced after contrast administration and imaging characteristics were consistent with fibrous dysplasia.

The patient was referred to our center for further evaluation and treatment. Based on the fact that the tumor could represent a symptomatic craniopharyngioma, resection was recommended. Although an underdeveloped or obstructed sphenoid sinus has historically been a relative contraindication to transphenoidal surgery, it was determined that an endoscopic transnasal approach would facilitate removal of the fibrous dyplasia involving the sphenoid sinus and also would be the best approach for resection of the sellar/suprasellar tumor.

\section{Intraoperative course}

Under general anesthesia, the patient's nose was infiltrated with $5 \mathrm{ml}$ of $2 \%$ Xylocaine with 1:100,000 epinephrine. Topicalizing cocaine pledgets were placed intranasally. A neuronavigation system with CT and MRI image fusion capability was used.

First, the middle turbinates were displaced laterally and outfractured to create an adequate operating pathway. The right sphenoid os was identified and opened. There was no identifiable sphenoid os on the left side as the sphenoid sinus in this region was completely replaced by abnormal bone. A posterior nasal septectomy was performed to allow binaral access to the posterior nasal cavity and sphenoid sinus. A Jansen-Middleton double action rongeur (C-Med Surgical, Inc.; Pompton Lakes, NJ) was used to resect the sphenoid rostrum. The bone

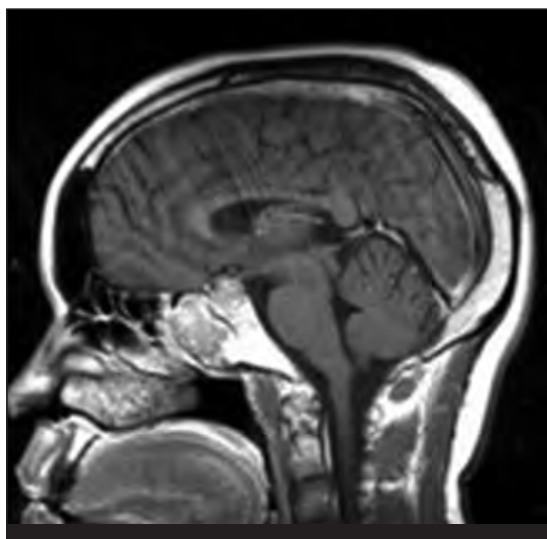

Figure 1

Preoperative sagittal T1-weighted MRI with gadolinium showing an enhancing sellar mass along with a space-occupying lesion involving the sphenoid bone. 


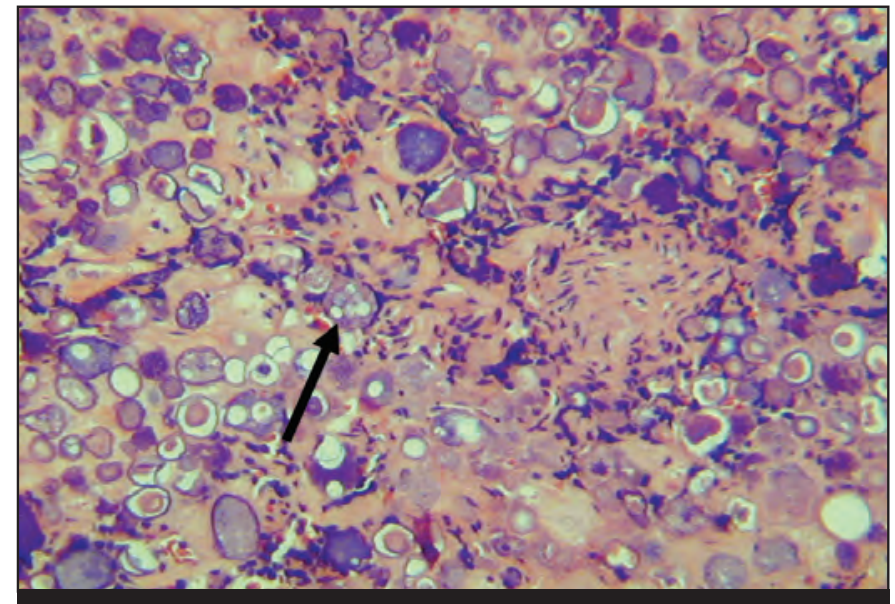

Figure 2

Photomicrograph demonstrating abundant microcalcifications (black arrow) within a pituitary adenoma. $\mathrm{H} \& \mathrm{E}$, original

magnification $\mathrm{x} 40$.

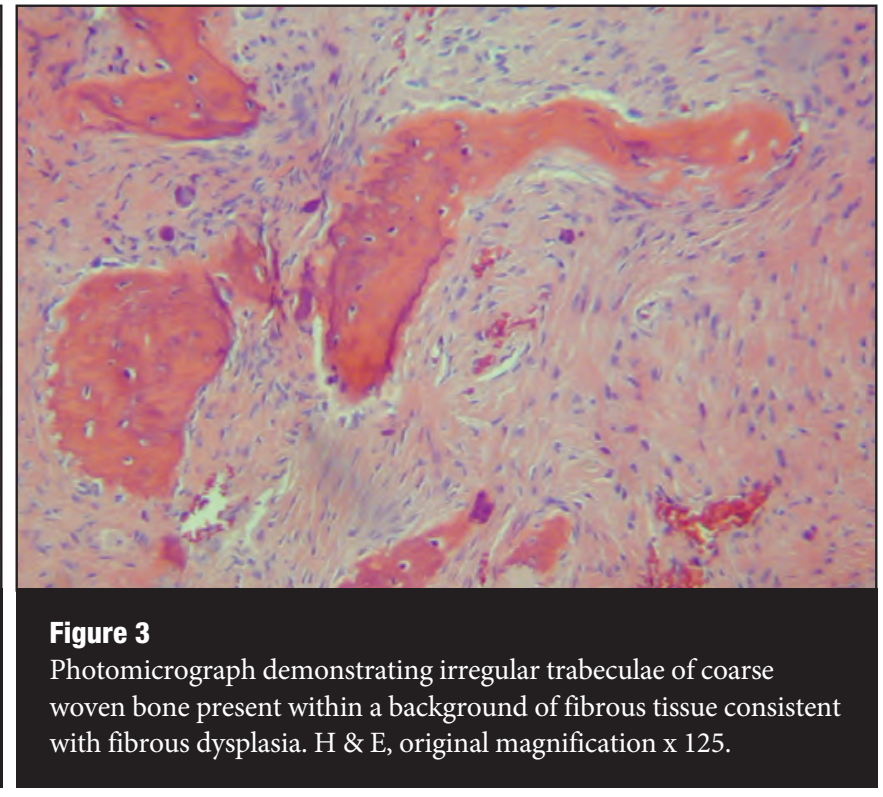

Figure 3

Photomicrograph demonstrating irregular trabeculae of coarse with fibrous dysplasia. H \& E, original magnification x 125 . was noted to be quite dense in areas and was grossly consistent with fibrous dysplasia. A Midas Rex drill (Medtronic, Inc.; Minneapolis, $\mathrm{MN}$ ) with a $2.5 \mathrm{~mm}$ matchstick cutting burr was used to perform drilling of the dense bone in the region of the sphenoid sinus, clivus, and laterally toward the left pterygoid. This provided endonasal access to the region of the sella.

Dense bone was encountered up to the cortical bone of the sella. Then, a $3 \mathrm{~mm}$ matchstick diamond drill was used to remove the cortical bone of the sella and expose the underlying dura. The dura of the sella was exposed from essentially the tuberculum sella down to the clivus and bilaterally toward the internal carotid arteries to allow adequate access to the suprasellar tumor.

The dura was then cauterized in a cruciate fashion using concentric suction bipolar electrocautery. A retractable endoscopic scalpel was then used to open the outer layer of the dura on the intracavernous sinus in a cruciate fashion. Bleeding from the intercavernous sinus was controlled with packing of the hemostatic agents FloSeal (Baxter International, Inc.; Deerfield, IL) and Surgicel (Johnson \& Johnson, Inc.; New Brunswick, NJ).

Next, the posterior wall of the intercavernous sinus was cauterized and opened allowing access to the intradural compartment, where a yellow-colored calcified tumor was identified. The more normal appearing pituitary gland was displaced toward the right aspect of the sella. Using microdissection, a plane was developed between the normal appearing pituitary gland and the tumor capsule. The tumor capsule was then opened sharply and internal tumor debulking was performed using pituitary ring curettes. Zero degree and 30 degree endoscopes facilitated direct visualization of the tumor and surrounding structures for extracapsular dissection of the tumor. Finally, the tumor capsule was dissected away from the overlying diaphragm sella and the dura in the region of the dorsum sella with careful preservation of these structures. A gross, total resection of the tumor was achieved. Valsalva maneuvers were performed to confirm the absence of any continued venous bleeding or cerebrospinal fluid leakage. Closure was performed using a sheet of DuraGen (Integra Life Sciences Corp.; Plainsboro, NJ) dural substitute placed in an inlay fashion within the dura followed by a dural substitute onlay graft. A synthetic polyetheylene glycol based dural sealant was used to complete the repair. Next, the middle turbinates were medialized and sheets of sterile absorbable gelatin film were placed lateral to the middle turbinates in order to keep the middle meatus from becoming obstructed. Also, sheets of gelatin film were placed medial to the inferior turbinates to prevent synechiae formation ans nasal obstruction. No fat graft or lumbar drain was necessary.

\section{Postoperative course}

Postoperative MRI imaging of the brain revealed complete resection of the pituitary tumor. There was no longer mass effect upon the optic chiasm. The patient did experience transient diabetes insipidus which resolved and did not require chronic treatment. The patient had complete resolution of his headache, resolution of his oculomotor abnormalities, and did not develop any new endocrinopathy. He was discharged to home on postoperative day three after an extra day in the hospital due to transient diabetes insipidus.

\section{Pathology}

Histologic sections of the pituitary mass showed small nests of cells situated in areas of dense mineralization (Figure 2). Numerous microcalcifications were noted. The small nests of tumor cells showed positive immunohistochemical staining for CAM 5.2, synaptophysin, chromogranin, and prolactin. They were negative for EMA and TSH, consistent with a calcified pituitary adenoma.

The sphenoid bone specimen contained irregular trabeculae of bone in a dense fibrous matrix. The bone spicules demonstrated a paucity of osteocytes and only scant osteoplastic activity was present, consistent with fibrous dysplasia (Figure 3).

\section{Discussion}

Fibrous dysplasia can occur in one (monostotic) or multiple (polyostotic) bones ${ }^{1,2}$. It has also been reported in association with other diseases, as in McCune-Albright syndrome ${ }^{3-6}$. Trabeculae of woven bone are contained in fluid-filled cysts embedded in a collagenous fibrous matrix, which contributes to the generalized hazy appearance of the bone on x-rays and CT scans ${ }^{7}$. The presence of fibrous dysplasia 
of the sphenoid bone in this patient presented a challenge to accessing the sella through an endonasal endoscopic approach.

Given the patient's preoperative complaints of headache, it was felt that the fibrous dysplasia could be responsible for these symptoms and therefore removing the fibrous dysplasia en route to the pituitary and suprasellar region could be beneficial. Given the imaging findings, and that the patient had galactorrhea, mild prolactinemia, and low testosterone level, it was felt that the sellar tumor was mostly likely a pituitary adenoma. Based on the fact that the tumor was partially calcified and suprasellar, a craniopharyngioma was also possible. Endoscopic resection of sellar and suprasellar tumors such as pituitary adenomas can result in a decreased length of stay, quicker recovery and fewer complications and was therefore the preferred approach ${ }^{8,9}$. Neuronavigation was essential in this case to allow for identification of critical landmarks, including the carotid arteries and the sella, which was obscured by the fibrous dysplasia.

Fibrous dysplasia is a benign tumor and therefore when it is asymptomatic it can usually be observed $^{7}$. Surgical intervention is indicated for symptomatic lesions causing intractablebone pain, severely reduced mobility, cosmetic deformity, or local pressure on vital structures ${ }^{3}$. In this case it was felt that fibrous dysplasia may have been responsible for the patient's headaches. Furthermore, removal of the fibrous dysplasia was necessary in this case to gain access to the pituitary tumor.

Removing fibrous dysplasia in this region runs the risk of injury to the skull base, carotid arteries, and optic nerves and chiasm. Multiple approaches to fibrous dysplasia of the sphenoid bone have been described, including endonasal, transfrontal and subcranial ${ }^{4,10}$. Isenberg was the first to describe the endoscopic approach for the diagnosis of fibrous dysplasia of the ethmoid $\operatorname{sinus}^{11}$. The current report is the first in the literature to describe an endoscopic approach to address fibrous dysplasia of the sphenoid bone.

Endoscopic endonasal removal of pituitary adenomas has been well described and is becoming the approach of choice at many institutions ${ }^{8-9,12}$. However endoscopic endonasal removal of fibrous dysplasia has not been widely described. With the advent of neuronavigation and ability to use CT, MRI and CT-MRI fusion, the approach to the sellar and suprasellar region even in the face of fibrous dysplasia or nonpneumatized sphenoid is feasible. We believe that osseous or fibro-osseous lesions involving the sphenoid are not necessarily a contraindication to endoscopic transsphenoidal surgery. In this case, the endonasal endoscopic approach allowed management of the fibrous dysplasia obstructing the sphenoid sinus facilitating access and gross total resection of the calcified pituitary adenoma.

\section{References}

1. Lichtenstein L. Polyostotic fibrous dysplasia. Arch Surg 1938;36:874-98.

2. Lichtenstein L, Jaffe HL. Fibrous dysplasia of bone. Archives of Pathology 1942;33:777-816.DiCaprio MR, Enneking WF. Fibrous dysplasia. Pathophysiology, evaluation, and treatment. J Bone Joint Surg Am 2005;87:1848-64.

3. Selmani Z, Aitasalo K, Ashammakhi N. Fibrous dysplasia of the sphenoid sinus and skull base presents in an adult with localized temporal headache. J Craniofac Surg 2004;15:261-3.

4. Parekh SG, Donthineni-Rao R, Ricchetti E, et al. Fibrous dysplasia. J Am Acad Orthop Surg 2004;12:305-13.

5. Bhansali A, Sharma BS, Sreenivasulu P, et al. Acromegaly with fibrous dysplasia: McCune-Albright Syndrome -- clinical studies in 3 cases and brief review of literature. Endocr I 2003;50:793-9.

6. Anand MKN. Fibrous dysplasia. In: wwwemedicinecom; 2006.

7. Jho HD, Carrau RL. Endoscopic endonasal transsphenoidal surgery: experience with 50 patients. J Neurosurg 1997;87:44-51.

8. de Divitiis E, Cappabianca P. Microscopic and endoscopic transsphenoidal surgery. Neurosurgery 2002;51:1527-9; author reply 9-30.

9. Lustig LR, Holliday MJ, McCarthy EF, et al. Fibrous dysplasia involving the skull base and temporal bone. Arch Otolaryngol Head Neck Surg 2001;127:1239-47.

10. Isenberg SF. Endoscopic approach to the diagnosis of fibrous dysplasia. Ear Nose Throat J 1994;73:926-7.

11. Sethi DS, Pillay PK. Endoscopic management of lesions of the sella turcica. J Laryngol Otol 1995; 109:956-62.

Corresponding Author:

Sanjay Yadla, MD

Department of Neurological Surgery

909 Walnut St, 3rd Floor

Philadelphia, PA 19107

Phone: 215-955-7000

Fax: Fax: 215-503-9170

Email: sanjay.yadla@jeffersonhospital.org

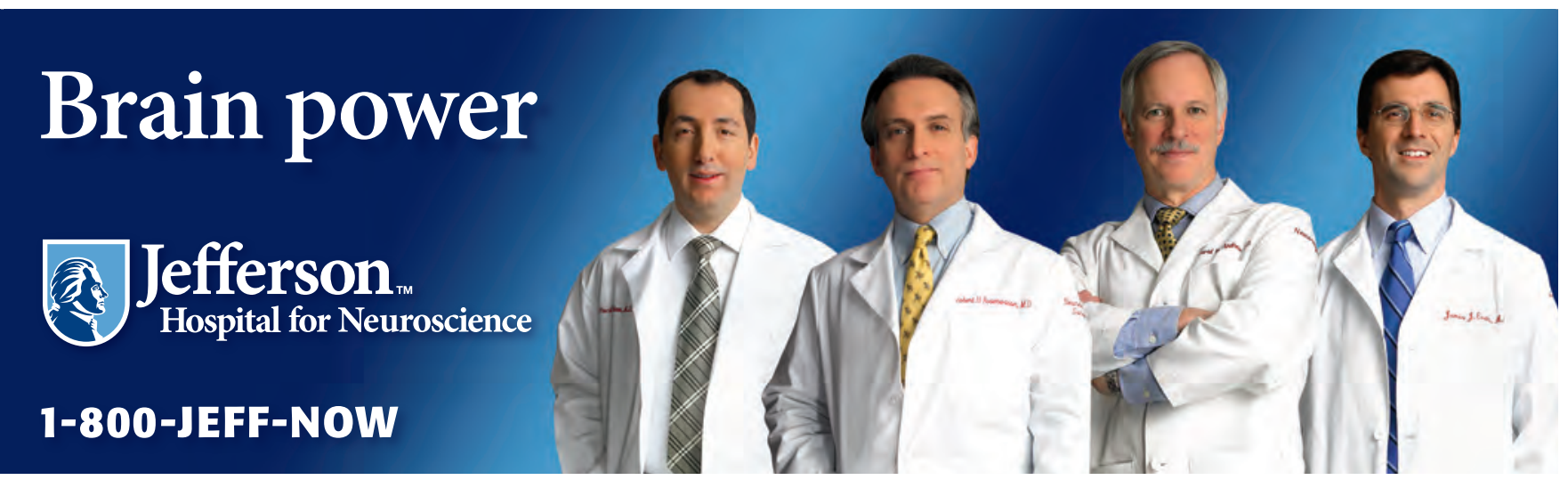

